\title{
A LINEAR NUMERICAL SCHEME FOR NONLINEAR BSDEs WITH UNIFORMLY CONTINUOUS COEFFICIENTS
}

\author{
OMID. S. FARD AND ALI. V. KAMYAD
}

Received 31 January 2004 and in revised form 23 June 2004

We attempt to present a new numerical approach to solve nonlinear backward stochastic differential equations. First, we present some definitions and theorems to obtain the condition, from which we can approximate the nonlinear term of the backward stochastic differential equation (BSDE) and we get a continuous piecewise linear BSDE corresponding to the original BSDE. We use the relationship between backward stochastic differential equations and stochastic controls by interpreting BSDEs as some stochastic optimal control problems to solve the approximated BSDE and we prove that the approximated solution converges to the exact solution of the original nonlinear BSDE.

\section{Introduction}

The backward stochastic differential equation (BSDE) theory and its applications have been a focus of interest in recent years. Consider the nonlinear BSDE

$$
\begin{gathered}
d x(t)=f(x(t), z(t), t) d t+z(t) d W(t), \\
x(T)=\xi
\end{gathered}
$$

where $\xi$ is a random variable that will become certain only at the terminal time $T$. This type of equation, at least in nonlinear case, was first introduced by Pardoux and Peng [14], who proved the existence and uniqueness of a solution under suitable assumptions on $f$

and $\xi$. Their aim was to give a probabilistic interpretation of a solution to a second-order quasilinear partial differential equation. The BSDE theory has found wide applications in partial differential equations, stochastic controls, and especially, mathematical finance.

For example, the Black-Scholes formula for option pricing can be recovered via a system of forward-backward stochastic differential equations (FBSDEs). In this case, the random terminal condition is related to the price of the underlying stock at a given terminal date. Unlike a (forward) SDE, the solution of a BSDE is a pair of adapted processes $(x(\cdot), z(\cdot))$. The additional term $z(\cdot)$ may be interpreted as a risk-adjustment factor and is required for the equation to have adapted solutions. This restriction of solutions to 
the class of adapted processes is necessary if the insights gained from the study of BSDEs are to be useful in applications. The adapted processes depend on the past and present information but do not rely on the future knowledge. This is normal virtually in all applications; for example, the replicating portfolio for a contingent claim may depend at any particular time on the past and present stock price but not, quite normally, on the future stock prices [15].

The still-standing problem in the theory of BSDEs is to find an implementable numerical method. Many efforts have been made in this direction as well. For example, in the Markovian case, Douglas et al. [7] established a numerical method for a class of forward-backward SDEs - a more general version of the BSDEs (1.1) — based on a fourstep scheme developed by Ma et al. [11]. Chevance, in his Ph.D. thesis [6], proposed a numerical method for BSDEs by using binomial approach to approximate the process $x$. In the non-Markovian case, where the terminal value $\xi$ is allowed to depend on the history of a forward diffusion $x$, Bally [3] presented a discretization scheme and obtained its rate of convergence. Recently, Briand et al. [5] and Ma et al. [10] also proposed some numerical methods for BSDEs with path-dependent terminal values.

Zhang and Zheng [17] suggested another method using PDE approach, in which high regularity of coefficients are required. Finally, Zhang, in his Ph.D. thesis [16], presented a numerical method for a class of BSDEs, whose terminal value $\xi$ takes the form $\phi(x)$, where $x$ is a diffusion process, and $\phi(\cdot)$ is a so-called $L^{\infty}$-Lipschitz functional.

On the other hand, Kohlmann and Zhou [9] explored the relationship between linear BSDEs and stochastic controls from a different angle by interpreting BSDEs as a stochastic linear-quadratic (LQ) control problem which can be solved explicitly. As mentioned in [9], "To be precise, note that in (1.1) the terminal value is specified while the initial value is left open. Nevertheless, if the equation has a solution, then the initial value cannot be chosen arbitrarily; rather it is uniquely determined by the solution and is hence part of the solution. Therefore, solving (1.1) amounts to the following statement: start with a proper initial condition and choose an appropriate diffusion term to hit the given value $\xi$ at the terminal. Then it will be very natural to modify the above statement and consider the following stochastic optional control problem. For the same dynamics of (1.1), starting with a given initial state $x_{0}$, choose a control $z(\cdot)$ so that the terminal state $x(T)$ stays as close to the given terminal value $\xi$ as possible. Note that since now the initial value $x_{0}$ is given a priori, one in general cannot expect that $x(T)$ will hit $\xi$ exactly by choosing certain $z(\cdot)$. Hence, it is reasonable to require that the difference between the two be minimized. Here, the "difference" may be measured by, say, the second moment of the algebraic difference between the two random variables. More interestingly, if we regard the initial state also as a decision variable, then the optimal state-control pair of the problem $(x(\cdot), z(\cdot))$ is exactly the solution of the original BSDE!”.

In this paper, we will try to develop this method for nonlinear BSDEs in such a way that the nonlinear coefficient of BSDE (1.1) can be approximated by a piecewise linear function and therefore we obtain a piecewise linear BSDE associated with (1.1). Then, we will prove that the solution of the obtained piecewise linear BSDE converges to the original solution of the nonlinear BSDE (1.1). Afterwards, we will find the optimal control problem associated with the obtained piecewise linear BSDE. 


\section{Problem formulation and preliminaries}

2.1. Notations. Throughout this paper $\left(\Omega, F, P,\left\{F_{t}\right\}_{t \geq 0}\right)$ is a fixed filtered complete probability space on which a standard $F_{t}$-adapted $m$-dimensional Brownian motion $W(t)=$ $\left(w^{1}(t), \ldots, w^{m}(t)\right)^{\prime}$ with $W(0)=0$, is defined. It is assumed that $F_{t}=\sigma\{W(t): s \leq t\}$. Let $X$ be a given Hilbert space. The set of $X$-valued continuous functions is denoted by $C([0, T] ; X)$. Suppose $\eta: \Omega \rightarrow \mathbb{R}^{n}$ is an $F_{t}$-random variable, we write $\eta \in L_{F}^{2}\left(\Omega ; \mathbb{R}^{n}\right)$, if $\eta$ is square integrable (i.e., $\left.E|\eta|^{2}<\infty\right)$. Now, consider the case when $f:[0, T] \times \Omega \rightarrow \mathbb{R}^{n}$ is an $\left\{F_{t}\right\}_{t \geq 0}$ adapted process. If $f(\cdot)$ is square integrable (i.e., $E \int_{0}^{T}|f(t)|^{2} d t<\infty$ ), we will write $f(\cdot) \in L_{F}^{2}\left(0, T ; \mathbb{R}^{n}\right)$. These definitions are general in the obvious way for the case when $f(\cdot)$ is $\mathbb{R}^{n \times m}$-valued.

Also, we make the following additional notations:

(i) $P^{\prime}$ denotes the transpose of any vector or matrix $P$;

(ii) $\langle a, b\rangle$ denotes the inner product of two vectors $a$ and $b$ (i.e., $\langle a, b\rangle=a \cdot b^{\prime}$ );

(iii) $S^{n}$ denotes the space of all $n \times n$ symmetric matrices;

(iv) $\hat{S}_{+}^{n}$ denotes the subspace of all positive definite matrices of $S^{n}$;

(v) $H^{2, k}$ denotes the set of $P$-measurable processes $V=\left\{V_{t}\right\}_{t \leq 1}$ with values in $\mathbb{R}^{k}$ such that $E \int_{0}^{1}\left|V_{s}\right|^{2} d s<\infty$;

(vi) $S^{2, k}$ denotes the set of continuous $P$-measurable processes $V=\left\{V_{t}\right\}_{t \leq 1}$ with values in $\mathbb{R}^{k}$ such that $E\left(\sup _{t \leq 1}\left|V_{t}\right|^{2}\right)<\infty$.

2.2. Existence and uniqueness of solution. Consider the following nonlinear BSDE:

$$
\begin{gathered}
d x(t)=f(x(t), z(t), t) d t-z(t) d W(t), \\
x(T)=\xi
\end{gathered}
$$

where $x(t) \in \mathbb{R}^{n}, z(t) \in \mathbb{R}^{n \times m}, f$ is a mapping from $\mathbb{R}^{n} \times \mathbb{R}^{n \times m} \times[0, T]$ to $\mathbb{R}^{n}$, and $\xi \in$ $L_{F}^{2}\left(\Omega ; \mathbb{R}^{n}\right)$.

Note that $f$ can be random, here, we assume that $f$ is a deterministic function.

Using the definition of the stochastic differential, (2.1) is equivalent to the following stochastic integral equation:

$$
x(t)=\xi-\int_{t}^{T} f(x(s), z(s), s) d t+\int_{t}^{T} z(s) d W(s) .
$$

We now give a precise definition of a solution to the BSDE.

Definition 2.1. A pair of stochastic processes

$$
\{x(t), z(t)\}_{0 \leq t \leq T} \in L_{F}^{2}\left([0, T] ; \mathbb{R}^{n}\right) \times L_{F}^{2}\left([0, T] ; \mathbb{R}^{n \times m}\right)
$$

is called an adapted solution of (2.1) if it has the following properties:

(i) $f(x(\cdot), z(\cdot), \cdot) \in L_{F}^{2}\left([0, T] ; \mathbb{R}^{n}\right)$,

(ii) equation (2.2) holds for every $t \in[0, T]$ with probability 1. 
A solution $\{x(t), z(t)\}$ is said to be unique if for any other solution $\{\bar{x}(t), \bar{z}(t)\}$, we have

$$
\begin{gathered}
P\{x(t)=\bar{x}(t) \forall 0 \leq t \leq T\}=1, \\
E \int_{0}^{T}|z(s)-\bar{z}(s)|^{2} d s=0 .
\end{gathered}
$$

Pardoux and Peng proved the following existence and uniqueness theorem [14].

Theorem 2.2. Assume that

$$
f(0,0, \cdot) \in L_{F}^{2}\left([0, T] ; \mathbb{R}^{n}\right) .
$$

Also, assume that there exists a positive constant $K>0$ such that

$$
|f(x, z, t)-f(\bar{x}, \bar{z}, t)|^{2} \leq K\left(|x-\bar{x}|^{2}+|z-\bar{z}|^{2}\right) \text { a.s. }
$$

for all $x, \bar{x} \in \mathbb{R}^{n}, z, \bar{z} \in \mathbb{R}^{n \times m}$, and $t \in[0, T]$, then there exists a unique solution $\{x(t)$, $z(t)\}$ to $(2.1)$ in $L_{F}^{2}\left([0, T] ; \mathbb{R}^{n}\right) \times L_{F}^{2}\left([0, T] ; \mathbb{R}^{n \times m}\right)$.

For the proof of Theorem 2.2, see [13, Chapter 7] or [14].

The uniform Lipschitz condition is too strong to be applied. In [8], Hamadène proved the existence and uniqueness of solution to (2.1) in weaker conditions. To explore the theorem, which was proved by Hamadène, we need to present some assumptions.

Assumption 2.3. The process $\{f(0,0, t)\}_{t \leq 1}$ belongs to $H^{2, n}$ and, for any $(x, z) \in \mathbb{R}^{n} \times$ $\mathbb{R}^{n \times m},\{f(x, z, t)\}_{t \leq 1}$ is $P$-measurable.

Let $\bar{\Phi}: \mathbb{R}^{+} \rightarrow \mathbb{R}^{+}$be a continuous function of at most linear growth such that, $\bar{\Phi}(0)=0$ and $\bar{\Phi}(y)>0$ for all $y>0$. We would like $\bar{\Phi}$ to satisfy the following assumption.

Assumption 2.4. $u^{\gamma}(0) \rightarrow 0$ as $\gamma \rightarrow 0$, where $u^{\gamma}$ is the unique solution on $[0,1]$ of the deterministic backward differential equation (DBE)

$$
u^{\gamma}(t)=\gamma+\int \bar{\Phi}\left(n \cdot u^{\gamma}(t)\right) d t \quad t \leq 1, \gamma>0
$$

This assumption implies, besides Assumption 2.3, the mapping $(x, z, t) \rightarrow f(x, z, t)$ satisfies the following.

Assumption 2.5. (i) $f$ is uniformly continuous in $x$ uniformly with respect to $(z, t)$, that is, there exists a continuous nondecreasing function $\Phi$ from $\mathbb{R}^{+}$into itself with at most linear growth and satisfying $\Phi(0)=0$ and $\Phi(y)>0$ for all $y>0$ such that

$$
\left|f(x, z, t)-f\left(x^{\prime}, z, t\right)\right| \leq \Phi\left(\left|x-x^{\prime}\right|\right) \quad \forall t, x, x^{\prime}, z \text { a.s. }
$$

moreover, $\Phi$ satisfies Assumption 2.4.

(ii) $f$ is uniformly continuous in $z$, that is, there exists a continuous function $\psi$ from $\mathbb{R}^{+}$into itself with at most linear growth and satisfying $\psi(0)=0$, such that

$$
\left|f(x, z, t)-f\left(x, z^{\prime}, t\right)\right| \leq \psi\left(\left|z-z^{\prime}\right|\right) \quad \forall t, x, z, z^{\prime} \text { a.s. }
$$


(iii) For $i=1, \ldots, n$, the $i$ th component $f_{i}$ of $f$ depends only on the $i$ th row of the matrix $z$.

Theorem 2.6. Suppose that Assumptions 2.3 and 2.5 hold. Then there exists a process $(x, z)$ in $S^{2, n} \times H^{2, n \times m}$ such that

$$
x(t)=\xi+\int_{t}^{1} f(x(s), z(s), s) d s-\int_{t}^{1} z(s) d W(s) \quad \forall t \leq 1 .
$$

For the proof of Theorem 2.6, see [8, proof of Theorem 3.1].

Assumption 2.7. (i) For all $t, x, x^{\prime}, z,\left|f(x, z, t)-f\left(x^{\prime}, z, t\right)\right| \leq \Phi\left(\left|x-x^{\prime}\right|\right)$, where $\Phi$ is continuous and nondecreasing, grows at most linearly and satisfies $\Phi(0)=0, \Phi(y)>0$, for all $y>0$, and $\int_{0^{+}}[\Phi(y)] d y=\infty$.

(ii) The function $z \rightarrow f(x, z, t)$ is Lipschitz uniformly with respect to $(x, t)$. In addition, for any $i=1, \ldots, n, f_{i}(x, z, t)$ the $i$ th component of $f$, depends only on the $i$ th row of $z$.

Now, we have the following result.

Theorem 2.8. Under Assumptions 2.3 and 2.7, the solution $(x, z)$ of the BSDE (2.10) associated with $(f, \xi)$ is unique.

For the proof of Theorem 2.8, see [8, proof of Theorem 4.1].

The following theorem, which was proved by Mao in [12], is the especial case of Theorem 2.8.

THEOREm 2.9. Assume that the following conditions are fulfilled:

(i) $f(x(\cdot), z(\cdot), \cdot) \in L_{F}^{2}\left([0, T] ; \mathbb{R}^{n}\right)$,

(ii) for all $x, \bar{x} \in \mathbb{R}^{n}, z, \bar{z} \in \mathbb{R}^{n \times m}$, and $t \in[0, T]$,

$$
|f(x, z, t)-f(\bar{x}, \bar{z}, t)|^{2} \leq \mathcal{K}\left(|x-\bar{x}|^{2}\right)+K\left(|z-\bar{z}|^{2}\right) \text { a.s. }
$$

where $K$ is a positive constant and $\mathscr{K}(\cdot)$ is a concave increasing function from $[0, \infty)$ to $[0, \infty)$ such that $\mathscr{K}(0)=0, \mathscr{K}(u)>0$ for $u>0$ and

$$
\int_{0^{+}} \frac{d u}{\mathscr{K}(u)}=\infty
$$

Then there exists a unique solution $\{x(t), z(t)\}$ to $(2.1)$ in $L_{F}^{2}\left([0, T] ; \mathbb{R}^{n}\right) \times L_{F}^{2}([0, T]$; $\left.\mathbb{R}^{n \times m}\right)$.

For the proof of Theorem 2.9, see [12] or [13, Chapter 7].

2.3. Regularities. In this subsection, we present two theorems, which guarantee the $p$ th moment $(p \geq 2)$ of the solution to (2.1) to be finite.

Theorem 2.10. Let $p \geq 2$ and $\xi \in L_{F_{T}}^{p}\left(\Omega ; \mathbb{R}^{n}\right)$. Let the following conditions hold:

(i) $f(x(\cdot), z(\cdot), \cdot) \in L_{F}^{2}\left([0, T] ; \mathbb{R}^{n}\right)$,

(ii) there exists a positive constant $K>0$ such that

$$
|f(x, z, t)-f(0,0, t)|^{2} \leq K\left(1+|x|^{2}+|z|^{2}\right) \text { a.s. }
$$


for all $x \in \mathbb{R}^{n}, z \in \mathbb{R}^{n \times m}$, and $t \in[0, T]$. Then the solution of (2.1) has the properties that

$$
\begin{gathered}
E|x(t)|^{p} \leq\left(E|\xi|^{p}+C_{1}\right) \exp (2 p T(4 K+1)) \quad \forall 0 \leq t \leq T, \\
E \int_{0}^{T}|x(t)|^{p-2}|z(t)|^{2} d t \leq \frac{4}{p}\left(E|\xi|^{p}+C_{1}\right)[1+\exp (2 p T(4 K+1))],
\end{gathered}
$$

where

$$
C_{1}=\frac{2}{p} \int_{0}^{T}\left[\frac{p}{8 K}\left(|f(0,0, s)|^{2}+K\right)+4 p(1+K)\right]^{p / 2} d s<\infty .
$$

Note that (2.11) implies (2.13).

For the proof of Theorem 2.10, see [13, Chapter 7, proof of Theorem 5.1, page 259].

Theorem 2.11. Let $p \geq 2$ and $\xi \in L_{F_{T}}^{p}\left(\Omega ; \mathbb{R}^{n}\right)$. Let (2.11) and (2.12) hold. Then the solution of (2.1) has the properties that

$$
E\left(\sup _{0 \leq t \leq T}|x(t)|^{p}\right)<\infty, \quad E\left(\int_{0}^{T}|z(t)|^{2} d t\right)^{p / 2}<\infty .
$$

For the proof of Theorem 2.11, see [13, Chapter 7, proof of Theorem 5.2, page 261].

\section{Continuous piecewise linear approximation}

In this section, we try to find a way to be able to approximate the function $f$ in (2.1) by a continuous piecewise linear function.

By using Chebyshev's inequality [2, Chapter 2, page 84] and Theorems 2.10 and 2.11, we can derive that, there exist two positive constants $M_{1}$ and $M_{2}$ such that

$$
\begin{array}{ll}
\forall t \in[0, T], \omega \in \Omega & |x(t)|^{2} \leq M_{1} \text { a.s., } \\
\forall t \in[0, T], \omega \in \Omega & |z(t)|^{2} \leq M_{2} \text { a.s. }
\end{array}
$$

Therefore, there exist the closed ball $B_{1}$ in $\mathbb{R}^{n}$ and the closed ball $B_{2}$ in $\mathbb{R}^{n \times m}$, respectively, for $x(\cdot)$ and $z(\cdot)$ such that

$$
\forall t \in[0, T], \omega \in \Omega, \quad x(t) \in B_{1}, \quad z(t) \in B_{2} \text { a.s. }
$$

Now, we define the closed set $B$ in $\mathbb{R}^{n} \times \mathbb{R}^{n \times m} \times[0, T]$ as follows:

$$
B=B_{1} \times B_{2} \times[0, T]
$$

The following lemma plays an important role in this section.

Lemma 3.1. Let $f: N \rightarrow M$ be a function from the metric space $N$ to another metric space $M$. Let also, $f$ be uniformly continuous on $A$ in $D_{f}$ (domain of $f$ ), and $M$ be a complete space. Then, there exists a uniformly continuous function $\bar{f}$ on $\bar{A}$ (closure of $A$ ), such that the restriction of $\bar{f}$ on $A$ is equal to the restriction of $f$ on $A\left(\right.$ i.e., $\left.\left.\left.\bar{f}\right|_{A} \equiv f\right|_{A}\right)$. 
For the proof of Lemma 3.1, see [1, Chapter 4].

Now, we can start the approximation process. First, we define the closed set $B_{c} \in \mathbb{R}^{n} \times$ $\mathbb{R}^{n \times m} \times[0, T]$ as follows:

$$
B_{c}=\overline{B \cap D_{f}}
$$

where $D_{f}$ is the domain of $f$ in (2.1). Conditions (2.6) and (2.10) imply that $f$ in (2.1) is a uniformly continuous function on $B_{c}$ and so, Lemma 3.1 holds for $f$. In other words, if $\bar{f}$ is the unique extension of $f$ on $B_{c}$, then $\bar{f}$ is uniformly continuous on $B_{c}$.

The next theorem implies that if we consider function $\bar{f}$ as a deterministic function, then for a given $\varepsilon>0$, we can find a uniform approximation of the function by the linear functions as $a_{1 k} x(t)+\sum_{j=1}^{m} a_{2 k}^{j} z_{j}(t)+a_{3 k}$, where $a_{1 k}, a_{2 k}^{j} \in \mathbb{R}^{n \times n}, j=1,2, \ldots, m, a_{3 k} \in \mathbb{R}^{n}$, and $z_{j}(\cdot)$ is the $j$ th column of $z(\cdot)$. Alternatively, for every $\varepsilon>0$ given, we can divide the set $B_{1}$ to the finite disjoint cells $I_{1}, I_{2}, \ldots, I_{q}, q=q(\varepsilon)$, such that if the function $h_{q(\varepsilon)}$ is defined as follows:

$$
\forall(x(t), z(t), t) \in I_{k}, h_{q(\varepsilon)}(x(t), z(t), t)=a_{1 k} x(t)+\sum_{j=1}^{m} a_{2 k}^{j} z_{j}(t)+a_{3 k}, k=1,2, \ldots, q,
$$

then, we have

$$
\left|h_{q(\varepsilon)}-\bar{f}\right|_{B_{c}}<\varepsilon
$$

where

$$
\left|h_{q(\varepsilon)}-\bar{f}\right|_{B_{c}}=\operatorname{esssup}\left\{\left|h_{q(\varepsilon)}(x(t), z(t), t)-\bar{f}(x(t), z(t), t)\right|:(x(t), z(t), t) \in B_{c}\right\} .
$$

THEOREM 3.2. Let $f$ be a continuous function, whose domain $D_{f}$ is a compact cell in $\mathbb{R}^{p}$ and whose values belong to $\mathbb{R}^{q}$. Then $f$ can be uniformly approximated on $D_{f}$ by continuous piecewise linear functions.

For the proof of Theorem 3.2, see [4, proof of Theorem 24.5, page 169].

Based on what we said, $\bar{f}(\cdot)$ satisfies the conditions of Theorem 3.2, therefore, since $f(\cdot)$ is the restriction of $\bar{f}(\cdot)$ on $B \cap D_{f}$, we can conclude that

$$
\left|h_{q(\varepsilon)}-f\right|_{B \cap D_{f}}<\varepsilon
$$

and it means that, we can approximate function $f(\cdot)$ in $(2.1)$ by a continuous piecewise linear function such $h_{q(\varepsilon)}(\cdot)$ on $B \cap D_{f}$, almost surely.

Hence, if we replace $h_{q(\varepsilon)}$ with coefficient $f$ in $(2.1)$, we have

$$
\begin{gathered}
d x(t) \cong h_{q(\varepsilon)}(x(t), z(t), t) d t-z(t) d W(t), \\
x(T)=\xi,
\end{gathered}
$$


and by definition $\left\{x_{q(\varepsilon)}(t), z_{q(\varepsilon)}(t)\right\}$ as the approximated solution to (2.1), we obtain a new BSDE as follows:

$$
\begin{gathered}
d x_{q(\varepsilon)}(t)=h_{q(\varepsilon)}\left(x_{q(\varepsilon)}(t), z_{q(\varepsilon)}(t), t\right) d t-z_{q(\varepsilon)}(t) d W(t), \\
x_{q(\varepsilon)}(T)=\xi .
\end{gathered}
$$

In the next section, we will show that $\left\{x_{q(\varepsilon)}(t), z_{q(\varepsilon)}(t)\right\}$ converges to $\{x(t), z(t)\}$ in three different cases.

\section{Convergence}

In this section, we prove the convergence of the approximated solution $\left\{x_{q(\varepsilon)}(t), z_{q(\varepsilon)}(t)\right\}$ to the exact solution to (2.1).

Theorem 4.1. Assume that the conditions of Theorems 2.6 and 2.8 are fulfilled. Also, assume that $\left\{x_{q(\varepsilon)}(t), z_{q(\varepsilon)}(t)\right\}$ to be the solution of (3.10), when $T=1$. Then the approximated solution $\left\{x_{q(\varepsilon)}(t), z_{q(\varepsilon)}(t)\right\}$ converges to $\{x(t), z(t)\}$ in the sense that

$$
\lim _{q \rightarrow \infty}\left|x(t)-x_{q(\varepsilon)}(t)\right|=0 \quad \forall t \in[0,1] .
$$

Proof. Let $\varepsilon>0$ be given. First, we prove that the process $x-x_{q}$ is uniformly bounded, that is, there exists a constant $\widetilde{C}$ such that $\left|x(t)-x_{q}(t)\right| \leq \widetilde{C}$, for all $t \leq 1$. Indeed, using Ito's formula we arrive, for all $t \leq 1$,

$$
\begin{aligned}
\left|x-x_{q}\right|^{2}+\int_{t}^{1}\left|z-z_{q}\right|^{2} d s= & 2 \int_{t}^{1}\left\langle x-x_{q}, f(x, z, s)-h_{q}\left(x_{q}, z_{q}, s\right)\right\rangle d s \\
& +2 \int_{t}^{1}\left\langle x-x_{q}, z-z_{q}\right\rangle d W(s) .
\end{aligned}
$$

On the other hand, let $f\left(x_{q}, z_{q}, s\right)-h_{q}\left(x_{q}, z_{q}, s\right) \equiv \varepsilon\left(x_{q}, z_{q}, s\right)$, again for the simplicity, we set $\varepsilon(s) \equiv \varepsilon\left(x_{q}, z_{q}, s\right)$, therefore

$$
\begin{aligned}
\left|x-x_{q}\right|^{2}+\int_{t}^{1}\left|z-z_{q}\right|^{2} d s \\
=2 \int_{t}^{1}\left\langle x-x_{q}, f(x, z, s)-f\left(x_{q}, z_{q}, s\right)+\varepsilon(s)\right\rangle d s+2 \int_{t}^{1}\left\langle x-x_{q}, z-z_{q}\right\rangle d W(s) \\
=2 \int_{t}^{1}\left\langle x-x_{q}, f(x, z, s)-f\left(x_{q}, z_{q}, s\right)\right\rangle d s+2 \int_{t}^{1}\left\langle x-x_{q}, \varepsilon(s)\right\rangle d s \\
\quad+2 \int_{t}^{1}\left\langle x-x_{q}, z-z_{q}\right\rangle d W(s) \\
\leq 2 \int_{t}^{1}\left|x-x_{q}\right| \Phi\left(\left|x-x_{q}\right|\right) d s+2 k \int_{t}^{1}\left|x-x_{q}\right|\left|z-z_{q}\right| d s \\
\quad+2 \int_{t}^{1}\left\langle x-x_{q}, \varepsilon(s)\right\rangle d s+2 \int_{t}^{1}\left\langle x-x_{q}, z-z_{q}\right\rangle d W(s)
\end{aligned}
$$

where $k$ is the Lipschitz constant. Since, for all $a, b \in \mathbb{R}$ and $\delta>0,|a \cdot b| \leq \delta a^{2}+\delta^{-1} b^{2}$ and the growth of $\Phi$ is at most linear, then $\Phi(|y|)^{2} \leq C_{2}\left(1+|y|^{2}\right)$ for all $y \in \mathbb{R}$, we can 
derive that

$$
\begin{aligned}
\left|x-x_{q}\right|^{2}+\int_{t}^{1}\left|z-z_{q}\right|^{2} d s \leq & C_{2} \int_{t}^{1}\left|x-x_{q}\right|^{2} d s+2 \int_{t}^{1}\left(1+\left|x-x_{q}\right|^{2}\right) d s \\
& +8 k \int_{t}^{1}\left|x-x_{q}\right|^{2} d s+\frac{1}{2} \int_{t}^{1}\left|z-z_{q}\right|^{2} d s \\
& +\frac{2}{C_{2}} \int_{t}^{1}|\varepsilon(s)|^{2} d s+2 \int_{t}^{1}\left\langle x-x_{q}, z-z_{q}\right\rangle d W(s) .
\end{aligned}
$$

On the other hand, since $(x, z)$ and $\left(x_{q}, z_{q}\right)$ belong to $S^{2, n} \times H^{2, n \times m}$, then using the Burkholder-Davis-Gundy inequality, we deduce that $\left\{\int_{0}^{t}\left\langle x-x_{q}, z-z_{q}\right\rangle d W(s)\right\}_{t \leq 1}$ is an $F_{t}$-martingale. We thus have

$$
\left|x-x_{q}\right|^{2} \leq C_{3}\left\{1+\varepsilon^{2}+\int_{t}^{1}\left|x-x_{q}\right|^{2} d s\right\}+\int_{t}^{1}\left\langle x-x_{q}, z-z_{q}\right\rangle d W(s) \quad t \leq 1,
$$

where $\varepsilon \geq \sup _{0 \leq t \leq T}|\varepsilon(t)|$.

Then, for any $r \geq t \geq 0$, we have

$$
E\left(\left|x(r)-x_{q}(r)\right|^{2} \mid F_{t}\right) \leq C_{3}\left\{1+\varepsilon^{2}+\int_{r}^{1} E\left(\left|x(s)-x_{q}(s)\right|^{2} \mid F_{t}\right) d s\right\} .
$$

Now by the Gronwall inequality we obtain $E\left(\left|x(r)-x_{q}(r)\right|^{2} \mid F_{t}\right) \leq \widetilde{C}$, which yields the desired result after taking $r=t$.

Now, we show the convergence of the approximated solution to the exact solution.

For any $i=1, \ldots, n$ and $t \leq 1$, we have

$$
x^{i}(t)-x_{q}^{i}(t)=\int_{t}^{1}\left(f^{i}\left(x, z^{i}, s\right)-h_{q}^{i}\left(x_{q}, z_{q}^{i}, s\right)\right) d s-\int_{t}^{1}\left(z^{i}-z_{q}^{i}\right) d W(s),
$$

where $x^{i}, x_{q}^{i}, f^{i}, h_{q}^{i}, z^{i}$, and $z_{q}^{i}$ are the $i$ th components and rows of, respectively, $x, x_{q}, f$, $h_{q}, z$ and $z_{q}$. Then, using Tanaka's formula, we obtain

$$
\begin{aligned}
\left|x^{i}-x_{q}^{i}\right|+2\left(\Lambda_{1}^{i}(0)-\Lambda_{t}^{i}(0)\right)= & \int_{t}^{1} \operatorname{sgn}\left(x^{i}-x_{q}^{i}\right)\left(f^{i}\left(x, z^{i}, s\right)-h_{q}^{i}\left(x_{q}, z_{q}^{i}, s\right)\right) d s \\
& -\int_{t}^{1} \operatorname{sgn}\left(x^{i}-x_{q}^{i}\right)\left(z^{i}-z_{q}^{i}\right) d W(s) \quad t \leq 1,
\end{aligned}
$$

where $\left\{\Lambda_{t}^{i}(0)\right\}_{t \leq 1}$ is the local time of $x^{i}-x_{q}^{i}$ at 0 .

Again, let $f\left(x_{q}, z_{q}, s\right)-h_{q}\left(x_{q}, z_{q}, s\right) \equiv \varepsilon\left(x_{q}, z_{q}, s\right)$, and we set $\varepsilon(s) \equiv \varepsilon\left(x_{q}, z_{q}, s\right)$, therefore

$$
\begin{aligned}
\left|x^{i}-x_{q}^{i}\right|+2\left(\Lambda_{1}^{i}(0)-\Lambda_{t}^{i}(0)\right)= & \int_{t}^{1} \operatorname{sgn}\left(x^{i}-x_{q}^{i}\right)\left(f^{i}\left(x, z^{i}, s\right)-f^{i}\left(x_{q}, z_{q}^{i}, s\right)\right) d s \\
& +\int_{t}^{1} \operatorname{sgn}\left(x^{i}-x_{q}^{i}\right) \varepsilon(s) d s \\
& -\int_{t}^{1} \operatorname{sgn}\left(x^{i}-x_{q}^{i}\right)\left(z^{i}-z_{q}^{i}\right) d W(s) \quad t \leq 1 .
\end{aligned}
$$


Now, let $\left\{a_{t}^{i}\right\}_{t \leq 1}$ be the following bounded and $F_{t}$-adapted process:

$$
a_{t}^{i}= \begin{cases}\frac{f^{i}\left(x_{q}, z^{i}, t\right)-f^{i}\left(x_{q}, z_{q}^{i}, t\right)}{z^{i}-z_{q}^{i}} & \text { if } z^{i}-z_{q}^{i} \neq 0, \\ 0 & \text { otherwise. }\end{cases}
$$

Then

$$
\begin{aligned}
\left|x^{i}-x_{q}^{i}\right| \leq & \int_{t}^{1} \operatorname{sgn}\left(x^{i}-x_{q}^{i}\right)\left(f^{i}\left(x, z^{i}, s\right)-f^{i}\left(x_{q}, z^{i}, s\right)\right) d s+\varepsilon^{2} \\
& -\int_{t}^{1} \operatorname{sgn}\left(x^{i}-x_{q}^{i}\right)\left(z^{i}-z_{q}^{i}\right) d \bar{W}(s) \quad t \leq 1,
\end{aligned}
$$

where $\varepsilon \geq \sup _{0 \leq t \leq T}|\varepsilon(t)|$ and $\bar{W}(t)=W(t)-\int_{0}^{t} a_{s}^{i} d s, t \leq 1$, is a Brownian motion under the probability $\bar{P}^{i}$ on $(\Omega, F)$ defined by $d \bar{P}^{i} / d P=\exp \left(\int_{0}^{1} a_{s}^{i} d W(s)-(1 / 2) \int_{0}^{1}\left|a_{s}^{i}\right|^{2} d s\right)$. We thus have

$$
\left|x^{i}-x_{q}^{i}\right| \leq \int_{t}^{1} \Phi\left(x^{i}-x_{q}^{i}\right) d s+\varepsilon^{2}-\int_{t}^{1} \operatorname{sgn}\left(x^{i}-x_{q}^{i}\right)\left(z^{i}-z_{q}^{i}\right) d \bar{W}(s) \quad t \leq 1 .
$$

Then, for all $r \geq t$,

$$
\bar{E}^{i}\left(\left|x^{i}(r)-x_{q}^{i}(r)\right| \mid F_{t}\right) \leq \int_{r}^{1} \bar{E}^{i}\left(\Phi\left(\left|x^{i}(s)-x_{q}^{i}(s)\right|\right) \mid F_{t}\right) d s+\varepsilon^{2},
$$

since $\int_{0}^{t} \operatorname{sgn}\left(x^{i}-x_{q}^{i}\right)\left(z^{i}-z_{q}^{i}\right) d \bar{W}(s), t \leq 1$ is an $F_{t}$-martingale (for more details see [8, proof of Theorem 3.1]).

Now, for $d \geq 0$, let $\Phi_{d}$ be a Lipschitz function from $\mathbb{R}$ into itself such that, for all $y \in \mathbb{R}^{+}, \Phi_{d}(y) \rightarrow \Phi(y)$ as $d \rightarrow \infty$ (see [8, proof of Proposition 2.2] for the existence of $\left.\Phi_{d}\right)$.

For $d \geq 0$ and $\delta>0$, let $v_{\delta}^{d}$ be the function such that

$$
v_{\delta}^{d}(t)=\delta+\int_{t}^{1} \Phi_{d}\left(n \cdot v_{\delta}^{d}(s)\right) d s \quad t \leq 1
$$

Since $\left\{\Phi_{d}\right\}_{d \geq 0}$ is a nonincreasing sequence, $v_{\delta}^{d+1} \leq v_{\delta}^{d}$ for any $d \geq 0$. This implies that the sequence $\left\{v_{\delta}^{d}\right\}_{d \geq 0}$ converges pointwise to a function $v_{\delta}: \mathbb{R}^{+} \rightarrow \mathbb{R}^{+}$, which satisfies

$$
v_{\delta}(t)=\delta+\int_{t}^{1} \Phi\left(n \cdot v_{\delta}(s)\right) d s \quad t \leq 1
$$

Now if $\delta \leq \lambda$ then $v_{\delta}^{d} \leq v_{\lambda}^{d}$ for any $d \geq 0$, and $v_{\delta} \leq v_{\lambda}$. It follows that $v_{\delta} \rightarrow \bar{v}$ as $\delta \rightarrow 0$, where for any $t \leq 1, \bar{v}_{t}=\int_{t}^{1} \Phi(n \cdot \bar{v}(s)) d s$, so that $\bar{v} \equiv 0$ (according to [8, Proposition 3.2]).

Now for $\delta, d$, and $k_{1} \geq 0$, let $v_{\delta}^{d, k_{1}}$ be the function defined recursively as follows:

$$
\begin{gathered}
v_{\delta}^{d, 0}=\tilde{C} \\
v_{\delta}^{d, k_{1}}(t)=\delta+\int_{t}^{1} \Phi_{d}\left(n \cdot v_{\delta}^{d, k_{1}-1}(s)\right) d s \quad k_{1} \geq 1, t \leq 1 .
\end{gathered}
$$


Since $\Phi_{d}$ is Lipschitz, $v_{\delta}^{d, k_{1}} \rightarrow v_{\delta}^{d}$ as $k_{1} \rightarrow+\infty$. On the other hand, it is easily seen by induction that for all $k_{1} \geq 0,\left|x^{i}(t)-x_{q}^{i}(t)\right| \leq v_{\delta}^{d, k_{1}}(t), t \leq 1, i=1, \ldots, n$. Indeed, for $k_{1}=0$, the formula holds. Suppose it also holds for some $k_{1}-1$, then

$$
\Phi\left(\left|x-x_{q}\right|\right) \leq \Phi\left(n \cdot v_{\delta}^{d, k_{1}-1}(t)\right) \leq \Phi_{d}\left(n \cdot v_{\delta}^{d, k_{1}-1}(t)\right) \quad \forall t \leq 1 .
$$

Now, using (4.13) and (4.16), we have

$$
\left|x^{i}-x_{q}^{i}\right| \leq v_{\delta}^{d, k_{1}}(t) \quad \forall t \leq 1, i=1, \ldots, n .
$$

Taking the limit as first $k_{1} \rightarrow \infty$, then $d \rightarrow \infty, \delta \rightarrow 0$, and finally $\varepsilon \rightarrow 0$ (as $q \rightarrow \infty$ ), we obtain

$$
\left|x^{i}-x_{q}^{i}\right|=0 \quad \forall t \leq 1
$$

\section{Metamorphosis}

In the previous section, we showed that the solution (3.10) converges to exact solution to (2.1); therefore, we can use the problem (3.10) instead of the problem (2.1).

By using the definition of $h_{q}(x(t), z(t), t)$, which is a continuous piecewise linear function, we can substitute $h_{q}(x(t), z(t), t)$ by $A_{q} x(t)+\sum_{j=1}^{m} B_{q j} z_{j}(t)+C_{q}$ for all $t \in[0, T]$, where $A_{q}, B_{q j} \in \mathbb{R}^{n \times n}$, and $C_{q} \in \mathbb{R}^{n}$.

Hence, we have

$$
\begin{gathered}
d x(t)=\left[A_{q} x(t)+\sum_{j=1}^{m} B_{q j} z_{j}(t)+C_{q}\right] d t-\sum_{j=1}^{m} z_{j}(t) d w^{j}(t) \quad t \in[0, T], \\
x(T)=\xi .
\end{gathered}
$$

On the other hand, it should be noted that (5.1) is the especial case of the following linear BSDE:

$$
\begin{gathered}
d x(t)=\left[A(t) x(t)+\sum_{j=1}^{m} B_{j}(t) z_{j}(t)+f(t)\right] d t-\sum_{j=1}^{m} z_{j}(t) d w^{j}(t) \quad t \in[0, T], \\
x(T)=\xi,
\end{gathered}
$$

where $x(t), \xi, z_{j}(t), f(t) \in \mathbb{R}^{n}$, and $A(t), B_{j}(t) \in \mathbb{R}^{n \times n}$. Also $A(t), B_{j}(t)$ are bounded deterministic functions and $f \in L_{F}^{2}\left(0, T ; \mathbb{R}^{n}\right)$.

Kohlmann and Zhou in [9] explored the relationship between linear BSDEs and stochastic control by interpreting linear BSDEs as some optimal problems.

Now, we consider the controlled system

$$
\begin{gathered}
d p(t)=\left[A_{q} p(t)+\sum_{j=1}^{m} B_{q j} u_{j}(t)+C_{q}\right] d t+\sum_{j=1}^{m} u_{j}(t) d w^{j}(t) \quad t \in[0, T], \\
p(0)=p_{0},
\end{gathered}
$$


where $p(t), p_{0}, u_{j}(t) \in \mathbb{R}^{n}$, and $A_{q}, B_{q j}$ are two bounded deterministic continuous piecewise linear functions. For a given $F_{T}$-measurable square integrable random variable $\xi$ (i.e., $\left.E|\xi|^{2}<\infty\right)$, the problem is to select an $F_{t}$-adapted control process $u(\cdot) \equiv\left(u_{1}(\cdot), \ldots\right.$, $\left.u_{m}(\cdot)\right) \in L_{F}^{2}\left(0, T ; \mathbb{R}^{n \times m}\right)$ so as to minimize the cost functional

$$
J_{q}\left(p_{0}, u(\cdot)\right)=E \frac{1}{2}|p(T)-\xi|^{2}
$$

To simplify the cost functional, it is normal to define

$$
y(t)=p(t)-E\left(\xi \mid F_{t}\right)
$$

Since $E\left(\xi \mid F_{t}\right)$ is an $F_{t}$-martingale and $F_{t}$ is generated by the standard Brownian motion $W(t)$, by the martingale representation theorem [13], there is $v(\cdot) \equiv\left(v_{1}(\cdot), \ldots, v_{m}(\cdot)\right) \in$ $L_{F}^{2}\left(0, T ; \mathbb{R}^{n \times m}\right)$ so that

$$
E\left(\xi \mid F_{t}\right)=E \xi+\sum_{j=1}^{m} \int_{0}^{T} v_{j}(s) d w^{j}(s) .
$$

By (5.3), (5.5), and (5.6), with the new state variable $y(\cdot)$ the controlled system becomes

$$
\begin{gathered}
d y(t)=\left[A_{q} y(t)+\sum_{j=1}^{m} B_{q j} u_{j}(t)+g_{q}\right] d t+\sum_{j=1}^{m}\left[u_{j}(t)-v_{j}(t)\right] d w^{j}(t) \quad t \in[0, T], \\
y(0)=x_{0}-E \xi \equiv y
\end{gathered}
$$

where $g_{q}=C_{q}+A_{q} E\left(\xi \mid F_{t}\right)$, and the cost functional reduces to $J_{q}(y, u(\cdot))=E(1 / 2)|y(T)|^{2}$.

Notice that the above problem is a stochastic LQ control problem.

Now, by considering the defined notations, we derive that

$$
\begin{gathered}
\operatorname{Min}_{q}(y, u(\cdot))=E \frac{1}{2}|y(T)|^{2} \\
\text { s.t. } d y(t)=\left[A_{q} y(t)+\sum_{j=1}^{m} B_{q j} u_{j}(t)+g_{q}\right] d t+\sum_{j=1}^{m}\left[u_{j}(t)-v_{j}(t)\right] d w^{j}(t), \\
y(0)=y .
\end{gathered}
$$

Here, we introduce the following stochastic Riccati equation (SRE):

$$
\begin{gathered}
\dot{P}_{q}+P_{q} A_{q}+A_{q}^{\prime} P_{q}-\sum_{j=1}^{m} P_{q} B_{q j} P_{q}^{-1} B_{q j} P_{q}=0, \\
P_{q}(T)=I, \\
P_{q}(t)>0 \quad \forall t \in[0, T],
\end{gathered}
$$


along with a BSDE

$$
\begin{gathered}
d \varphi(t)=-\left[\left(A_{q}^{\prime}-\sum_{j=1}^{m} P_{q} B_{q j} P_{q}^{-1} B_{q j}^{\prime}\right) \varphi-\sum_{j=1}^{m} P_{q} B_{q j} P_{q}^{-1} \beta_{j}\right. \\
\left.+P_{q}\left(g_{q}+\sum_{j=1}^{m} B_{q j} v_{j}\right)\right](t) d t+\sum_{j=1}^{m} \beta_{j}(t) d w^{j}(t), \\
\varphi(T)=0 .
\end{gathered}
$$

Note that the SRE (5.9) is different from the traditional Riccati equation in that (5.9) involves the inverse of the unknown. Moreover, the third constraint of (5.9) must also be satisfied by any solution. In general, an equation like (5.9) does not automatically admit a solution. (The solvability of this equation is considered by Kohlmann and Zhou in [9, Section 4].) Besides, if (5.9) has a solution $P_{q}(\cdot) \in C\left([0, T] ; \mathbb{R}^{n \times n}\right)$, then (5.10) must admit an $F_{t}$-adapted solution $\left(\varphi(\cdot), \beta_{j}(\cdot), j=1, \ldots, m\right)$ as $(5.10)$ is a piecewise linear BSDE with bounded coefficients and square integrable nonhomogeneous terms. (For more details, see [15].)

The two following theorems are the especial cases of [9, Theorems 3.1 and 3.2], which were proved by Kohlmann and Zhou.

Theorem 5.1. If (5.9) and (5.10) admit solutions $P_{q} \in C\left([0, T] ; \hat{S}_{+}^{n}\right)$ and $\left(\varphi(\cdot), \beta_{j}(\cdot), j=\right.$ $1, \ldots, m) \in L_{F}^{2}\left(0, T ; \mathbb{R}^{n}\right) \times L_{F}^{2}\left(0, T ; \mathbb{R}^{n \times m}\right)$, respectively, then the problem (5.8) has an optimal feedback control $u^{*}(\cdot) \equiv\left(u_{1}^{*}(\cdot), \ldots, u_{j}^{*}(\cdot)\right)$, where

$$
u_{j}^{*}(t)=-P_{q}^{-1}(t) B_{q j}^{\prime}\left[P_{q}(t) y^{*}(t)+\varphi(t)\right]-P_{q}^{-1}(t) \beta_{j}(t)+v_{j}^{\prime}(t), \quad j=1, \ldots, m .
$$

Moreover, the optimal cost value under the above control is

$$
\begin{aligned}
J_{q}^{*}(y)= & \frac{1}{2} y^{\prime} P(0) y+y^{\prime} \varphi(0) \\
+\frac{1}{2} E \int_{0}^{T}[ & 2 \varphi^{\prime} g_{q}-2 \sum_{j=1}^{m} \beta_{j} v_{j}+\sum_{j=1}^{m} v_{j}^{\prime} P v_{j} \\
& \left.-\sum_{j=1}^{m}\left(P_{q}^{-1} B_{q j}^{\prime} \varphi+P_{q}^{-1} \beta_{j}-v_{j}\right)^{\prime} P\left(P_{q}^{-1} B_{j}^{\prime} \varphi+P_{q}^{-1} \beta_{j}-v_{j}\right)\right](t) d t .
\end{aligned}
$$

For the proof of Theorem 5.1, see [9, proof of Theorem 3.1].

THEOREM 5.2. Under the same assumptions of Theorem 5.1, the control problem (5.3)-(5.4) has an optimal feedback control $u^{*}(\cdot) \equiv\left(u_{1}^{*}(\cdot), \ldots, u_{j}^{*}(\cdot)\right)$, where

$$
u_{j}^{*}(t)=-P_{q}^{-1}(t) B_{q j}^{\prime} P_{q}(t)\left[p^{*}(t)-x_{q}(t)\right]-z_{q j}(t) \quad j=1, \ldots, m,
$$


where $\left(x_{q}(\cdot), z_{q j}(\cdot), j=1, \ldots, m\right) \in L_{F}^{2}\left(0, T ; \mathbb{R}^{n}\right) \times L_{F}^{2}\left(0, T ; \mathbb{R}^{n \times m}\right)$ is the unique $F_{t}$-adapted solution of the following BSDE:

$$
\begin{gathered}
d x(t)=\left[A_{q} x(t)+\sum_{j=1}^{m} B_{q j} z_{j}(t)+C_{q}\right] d t-\sum_{j=1}^{m} z_{j}(t) d w^{j}(t) \quad t \in[0, T], \\
x(T)=\xi .
\end{gathered}
$$

Notice that (5.14) yields

$$
\begin{gathered}
x_{q}(t)=E\left(\xi \mid F_{t}\right)-P_{q}(t)^{-1} \varphi(t), \\
z_{q j}(t)=v_{j}(t)-P_{q}(t)^{-1} \beta_{j}(t) \quad j=1, \ldots, m .
\end{gathered}
$$

For the proof of Theorem 5.2, see [9, proof of Theorem 3.2].

Remark 5.3. Equation (5.12) also gives the optimal cost functional value as a function of the initial value $y \equiv p_{0}-E \xi$, which turns out to be quadratic. If the controller has the choice of selecting the initial value $y$ so as to minimize $J_{q}^{*}(y)$, then the "best" initial value would be obtained by setting $\left.(d / d y) J_{q}^{*}(y)\right|_{y=y^{*}}=0$. This yields $y^{*}=-P_{q}(0)^{-1} \varphi(0)$. Returning to the original variable $p_{0}$, we get that the best initial value for $p(\cdot)$ will be

$$
p_{0}^{*}=y^{*}+E \xi=-P_{q}(0)^{-1} \varphi(0)+E \xi=x(0)
$$

where the last equality is due to (5.15). This certainly makes perfect sense, as it implies that one should choose the initial value $x_{q}(0)$ so as to minimize the difference between the terminal state value and the given value $\xi$. (Of course, in this case the minimum difference is zero since starting with $x_{q}(0)$ one can hit $\xi$ exactly at the end, by the BSDE theory.) Therefore, the solution pair $\left(x_{q}(\cdot), z_{q}(\cdot)\right)$ of the BSDE (5.14) may be regarded as the optimal state-control pair of minimizing $J_{q}\left(p_{0}, u(\cdot)\right)$ (as given by $\left.(5.4)\right)$ over $\left(p_{0}, u(\cdot)\right)$ subject to the dynamics of (5.3). This gives an interpretation of $\left(x_{q}(\cdot), z_{q}(\cdot)\right)$ via a stochastic control problem. In this perspective, if a BSDE does not have an adapted solution (e.g., when the underlying filtration is not generated by the Brownian motion involvement), we may still define a "pseudosolution" via the corresponding stochastic control problem.

Remark 5.4. Under the optimal feedback (5.13), the optimal trajectory $p^{*}(\cdot)$ evolves as

$$
\begin{gathered}
d p^{*}(t)=\left\{\left[A_{q}-\sum_{j=1}^{m} B_{q j} P_{q}^{-1} B_{q j}^{\prime} P_{q}\right] p^{*}+\sum_{j=1}^{m} B_{q j} P_{q}^{-1} B_{q j}^{\prime} P_{q} x_{q}+C_{q}+\sum_{j=1}^{m} B_{q j} z_{q j}\right\}(t) d t \\
+\sum_{j=1}^{m}\left[-P^{-1} B_{q j}^{\prime} P p_{q}^{*}+P_{q}^{-1} B_{q j}^{\prime} P_{q} x_{q}+z_{q}\right](t) d w^{j}(t), \\
p^{*}(0)=p_{0} .
\end{gathered}
$$




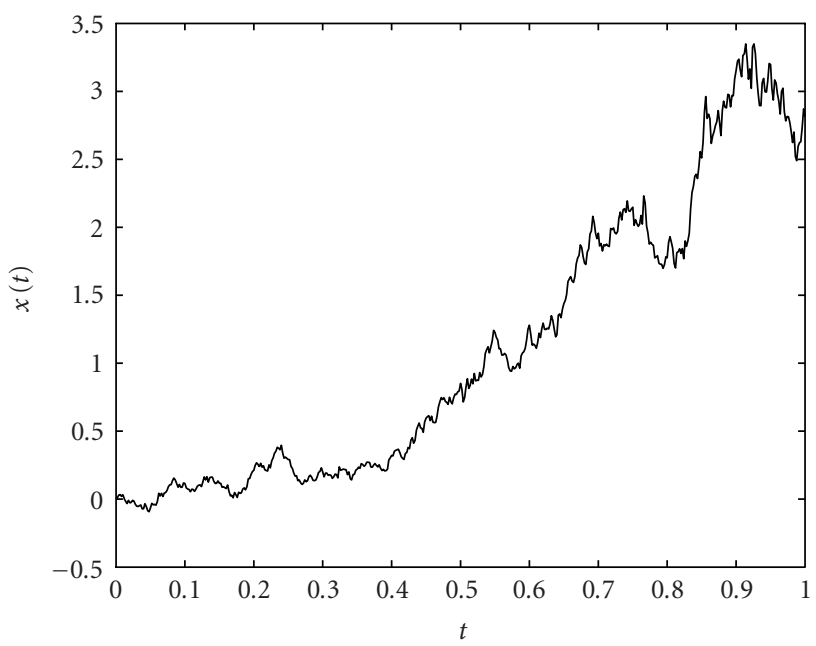

Figure 6.1

Moreover, the difference $\Delta(t)=p^{*}(t)-x_{q}(t)$ satisfies

$$
\begin{gathered}
d \Delta(t)=\left[A_{q}-\sum_{j=1}^{m} B_{q j} P_{q}^{-1} B_{q j}^{\prime} P_{q}\right](t) d t-\sum_{j=1}^{m}\left[-P_{q}^{-1} B_{q j}^{\prime} P_{q}\right](t) \Delta(t) d w^{j}(t), \\
\Delta(0)=p_{0}-x_{q}(0) .
\end{gathered}
$$

Notice that $\Delta(\cdot)$ satisfies a homogeneous linear SDE, and hence must be identically zero if the initial value is zero, namely, if $p_{0}=x_{q}(0)$. In this case, by (5.13), the optimal control is $u_{j}^{*}(t)=z_{q j}(t)$. This is exactly in line with the observation in Remark 5.3. (See [9].)

\section{Numerical results}

In this section, we use the results of previous sections to solve a nonlinear BSDE as (1.1) in two different cases.

Example 6.1. In this example, we set $f(x, z, t)=\sqrt{x(t)}+z(t), \xi=3$ and $T=1$, then we have the following nonlinear BSDE:

$$
\begin{gathered}
d x(t)=[\sqrt{x(t)}+z(t)] d t+z(t) d W(t), \\
x(1)=3 .
\end{gathered}
$$

First, we approximate the nonlinear term (i.e., $f(x, z, t)=\sqrt{x(t)}+z(t))$ by a continuous piecewise linear function as $A_{q} x(t)+z(t)$. We define a stochastic optimal control problem 


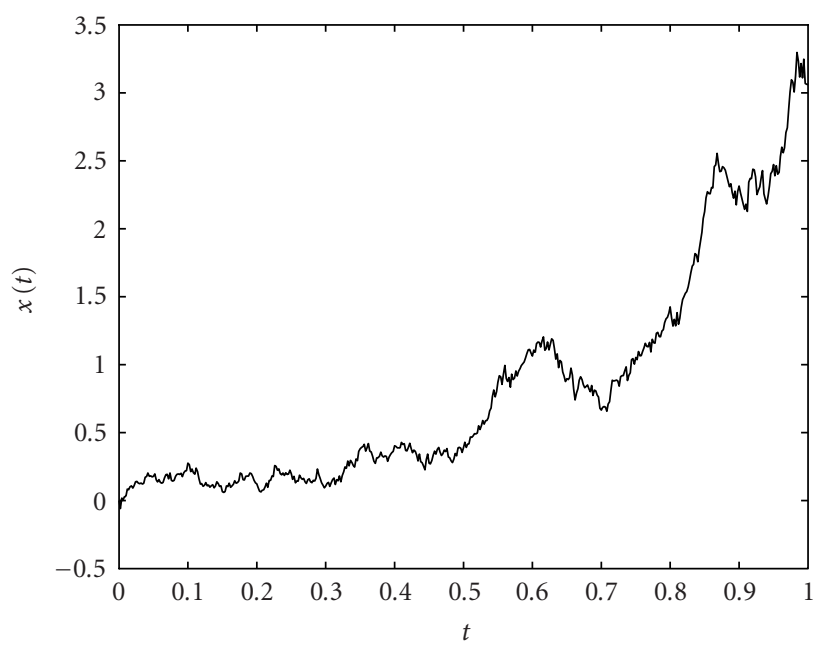

Figure 6.2

corresponding with the original problem as below:

$$
\begin{gathered}
J_{q}\left(p_{0}, u(\cdot)\right)=E \frac{1}{2}|p(1)-3|^{2} \\
\text { s.t. } d p(t)=\left[A_{q} p(t)+u(t)\right] d t+u(t) d W \quad t \in[0,1], \\
p(0)=p_{0} .
\end{gathered}
$$

In this example, we set $p_{0}=0$. By solving the stochastic optimal control problem, we obtain a sample path with $x_{q}^{*}(1)=2.922$. In Figure 6.1, we plot the obtained result.

Example 6.2. In this example, again we set $f(x, z, t)=\sqrt{x(t)}+z(t), \xi=3$ and $T=1$, then we have the same nonlinear BSDE in Example 6.1. But in this example we consider $p_{0}$ as a decision variable. By solving the stochastic optimal control problem, we obtain a sample path with $x_{q}^{*}(0)=-0.058$ and $x_{q}^{*}(1)=3.060$. Numerical result is in Figure 6.2.

\section{Acknowledgments}

The authors wish to thank S. Hamadène, N. Ghal-eh and N. Tavallaee for inspiring discussions. Also, the authors would like to thank the referee for his/her careful reading of the manuscript and his/her constructive comments that led to an improved version of the paper.

\section{References}

[1] T. M. Apostol, Mathematical Analysis, 2nd ed., Addison-Wesley Publishing, Massachusetts, 1974.

[2] R. B. Ash, Real Analysis and Probability, Academic Press, New York, 1972.

[3] V. Bally, An approximation scheme for BSDEs and applications to control and nonlinear PDEs, preprint, 1995. 
[4] R. G. Bartle, The Elements of Real Analysis, 2nd ed., John Wiley \& Sons, New York, 1976.

[5] P. Briand, B. Delyon, and J. Mémin, Donsker-type theorem for BSDEs, Electron. Comm. Probab. 6 (2001), 1-14.

[6] D. Chevance, Resolution numerique des èquations dioeerentielles stochastiques retrogrades, Ph.D. thesis, Université de Provence, Provence, 1997.

[7] J. Douglas Jr., J. Ma, and P. Protter, Numerical methods for forward-backward stochastic differential equations, Ann. Appl. Probab. 6 (1996), no. 3, 940-968.

[8] S. Hamadène, Multidimensional backward stochastic differential equations with uniformly continuous coefficients, Bernoulli 9 (2003), no. 3, 517-534.

[9] M. Kohlmann and X. Y. Zhou, Relationship between backward stochastic differential equations and stochastic controls: a linear-quadratic approach, SIAM J. Control Optim. 38 (2000), no. 5, 1392-1407.

[10] J. Ma, P. Protter, J. San Martín, and S. Torres, Numerical method for backward stochastic differential equations, Ann. Appl. Probab. 12 (2002), no. 1, 302-316.

[11] J. Ma, P. Protter, and J. M. Yong, Solving forward-backward stochastic differential equations explicitly — a four step scheme, Probab. Theory Related Fields 98 (1994), no. 3, 339-359.

[12] X. Mao, Adapted solutions of backward stochastic differential equations with non-Lipschitz coeffcients, Stochastic Process. Appl. 58 (1995), no. 2, 281-292.

[13] Stochastic Differential Equations and Their Applications, Horwood Publishing Series in Mathematics \& Applications, Horwood Publishing, Chichester, 1997.

[14] É. Pardoux and S. G. Peng, Adapted solution of a backward stochastic differential equation, Systems Control Lett. 14 (1990), no. 1, 55-61.

[15] J. Yong and X. Y. Zhou, Stochastic Controls. Hamiltonian Systems and HJB Equations, Applications of Mathematics, vol. 43, Springer-Verlag, New York, 1999.

[16] J. Zhang, Some fine properties of backward stochastic differential equations, Ph.D. thesis, Purdue University, Indiana, 2001.

[17] Y. Zhang and W. Zheng, Discretizing a backward stochastic differential equation, Int. J. Math. Math. Sci. 32 (2002), no. 2, 103-116.

Omid. S. Fard: Department of Mathematics, Damghan University of Basic Sciences, Damghan, Iran

E-mail address: osfard@dubs.ac.ir

Ali. V. Kamyad: Department of Mathematics, Ferdowsi University of Mashhad, Mashhad, Iran

E-mail address: kamyad@math.um.ac.ir 


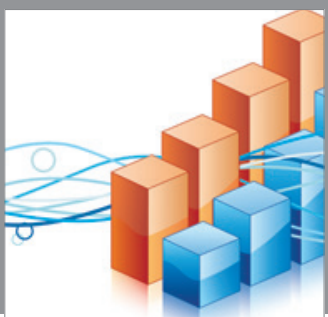

Advances in

Operations Research

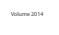

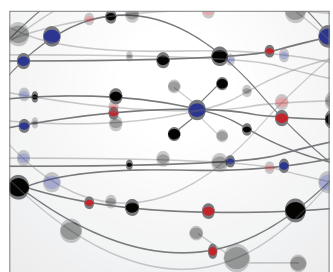

\section{The Scientific} World Journal
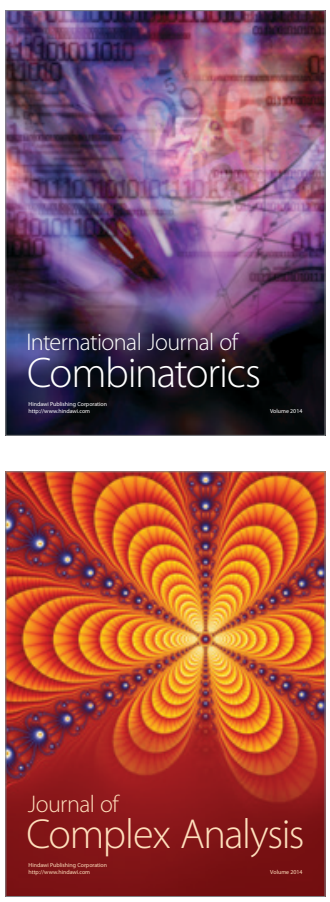

International Journal of

Mathematics and

Mathematical

Sciences
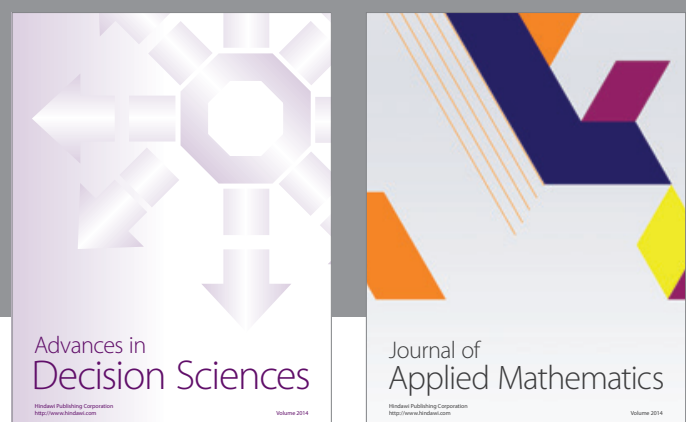

Journal of

Applied Mathematics
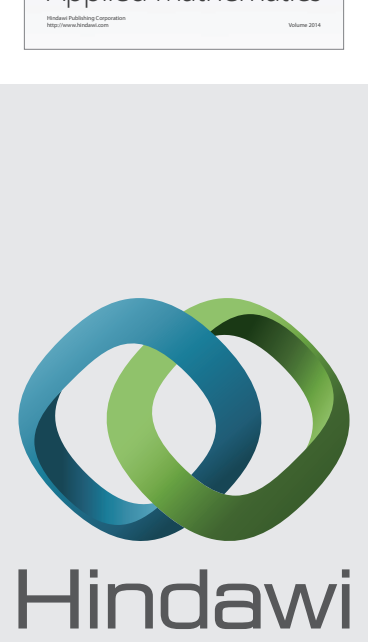

Submit your manuscripts at http://www.hindawi.com
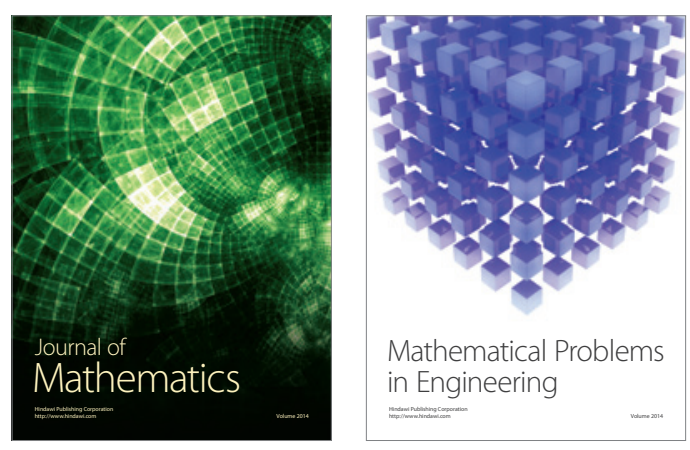

Mathematical Problems in Engineering
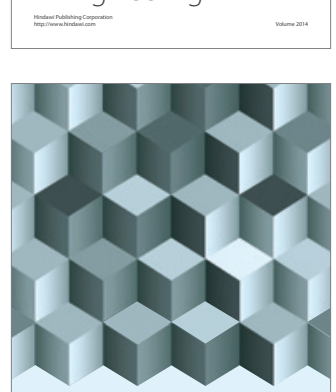

Journal of

Function Spaces
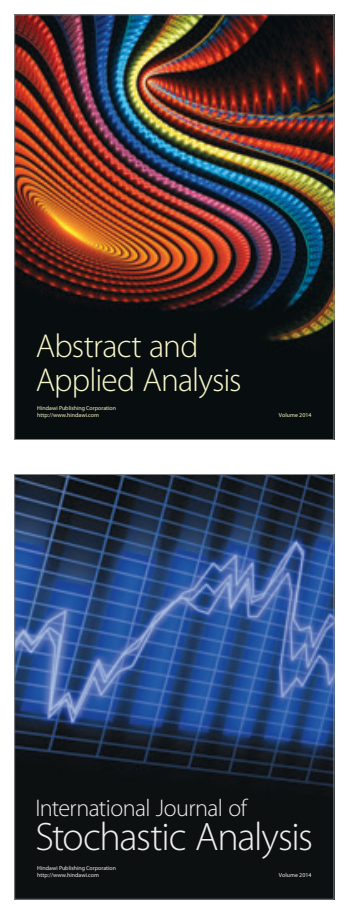

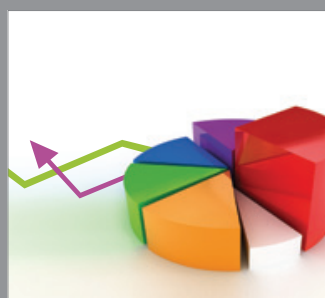

ournal of

Probability and Statistics

Promensencen
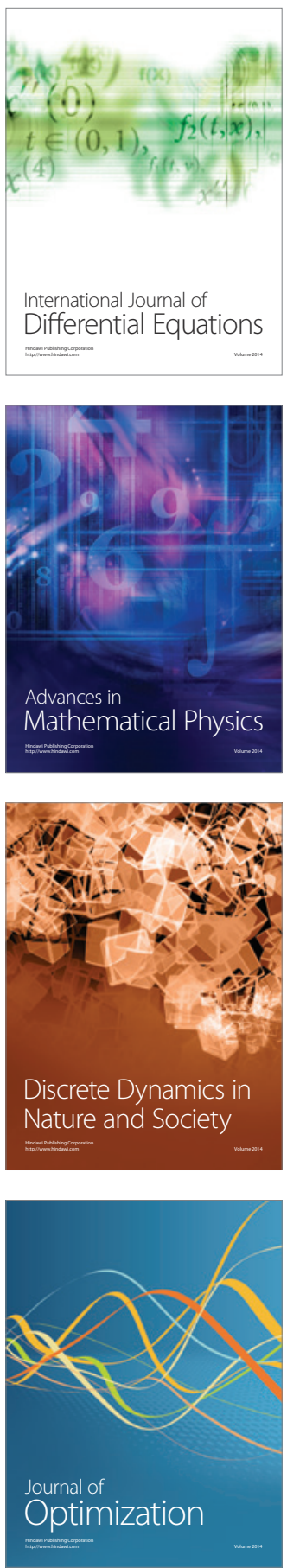